variable feedback," J. Dynam. Syst., Measurements, Contr., vol. pp. 323-329, 1972.

[29] P. K. Sinha, "State feedback decoupling of nonlinear systems," IEEE Trans. Automat. Contr., vol. AC-22, pp. 487-489, 1977.

[30] S. H. Wang, "Design of precompensator for decoupling problem," Electron. Lett., vol. 6, pp. 739-741, 1970 .

[31] W. M. Wonham and A. S. Morse, "Status of noninteracting control," IEEE Trans. Automat. Contr., vol. AC-16, pp. 568-581, 1971.

[32] L. M. Silverman, "Inversion of multivariable linear systems," IEEE Trans. Automat. Contr., vol. AC-14, pp. 270-276, 1969.

[33] J. Descusse and C. H. Moog, "Dynamic decoupling for right-invertible nonlinear systems," Syst. Contr. Lett., vol. 8, pp. 345-350, 1987.

[34] H. Nijmeijer and W. Respondek, "Decoupling via dynamic compensation for nonlinear control systems," in Proc. 25th CDC, Athens, Greece, 1986, pp. 192197.

[35] J. W. Grizzle, M. D. di Benedetto, and C. H. Moog, "Computing the differential output rank of a nonlinear system," in Proc. 26th CDC, Los Angeles, CA, 1987, pp. $142-145$.

[36] J. Descusse and C. H. Moog, "Some recent results on dynamic decoupling of nonlinear systems," Proc. SYROCO '85, Barcelona, Spain, 1985, pp. 283-286.

\section{Design for Noninteracting Decomposition of Nonlinear Systems}

\section{DAIZHAN CHENG}

Abstract-This note tackles the general input-output noninteracting decomposition problem of nonlinear systems. Under less regularity assumptions we give an alternative proof of the same necessary and sufficient conditions as in [4]. Our result gives an algorithm which constructs the feedback law $\alpha$ and $\beta$ explicitly. Finally, we prove that the decomposed form is a canonical form.

\section{INTRODUCTION}

Consider an affine nonlinear system

$$
\begin{gathered}
\dot{x}=f(x)+\sum_{i=1}^{m} g_{i}(x) u_{i} \\
y=h(x)
\end{gathered}
$$

where $x \in M ; f(x), g_{i}(x) \in V(M) ; h: M \rightarrow N$ is a $C^{\infty}$ mapping: $M$ and $N$ are $C^{\infty}$ manifolds with dimensions $n$ and $r$, respectively. The inputoutput noninteracting decomposition problem (NDP) may be stated as follows. Given a partition of the outputs $y$, whether there exist a feedback control

$$
u=\alpha(x)+\beta(x) v
$$

and a partition of the controls $v$, such that each block of $v$ completely controls the corresponding block of $y$, and does not affect the other blocks of the outputs.

The NDP has been studied extensively and from various points of view. The discussion for linear systems is founded in [1], [2], etc.

Recently, the NDP of nonlinear systems has been studied in [3] and [4]. Reference [3] gives precise formulation and solves NDP for the single-input and block-output case by "controllability distribution" approach. Reference [4] proves the same results for the block-input and block-output case under more regularity assumptions by using the concept of zeros at infinity.

The main goal of this note is to give an alternative proof of the same result of [4] under less regularity assumptions as required in [3]. Our proof is constructive, thus it yields an algorithm. Using it, an input-output

Manuscript received September 30, 1987.

The author is with the Department of Mathematics, Texas Tech University, Lubbock, TX $79405-4319$

IEEE Log Number 8822745 decomposed form has been obtained. Finally, we prove that the decomposed form obtained is a canonical form.

For investigating the decoupling problem of linear systems, the geometric concepts of $(A, B)$-invariant subspaces and controllability subspaces play a very important role. In the geometric approach to nonlinear systems, the concept of $(A, B)$-invariant subspaces has been extended to that of $(f, g)$-invariant distributions [5], [6], and the concept of controllability subspaces has also been extended to that of controllability distributions [7], [8].

Since our discussion depends particularly upon the concept of $(f, g)$ invariance, we state the following definition which is slightly different from the original one given in [6].

For the sake of compactness, let $C_{m}^{\infty}(U)$ be the set of $m \times 1$ vectors with the entries in $C^{\infty}(U)$, and $G 1\left(m, C^{\infty}(U)\right)$ be the set of $m \times m$ nonsingular matrices with the entries in $C^{\infty}(U)$ too, where $U$ is an open subset of $M$.

Definition 1.1: A distribution $\Delta$ is said to be weakly $(f, g)$-invariant at $p \in M$ if there exists a neighborhood $U$ of $p$, such that on $U$

$$
\begin{gathered}
{[f, \Delta] \subset \Delta+G,} \\
{\left[g_{i}, \Delta\right] \subset \Delta+G, \quad i=1, \cdots, m}
\end{gathered}
$$

where $G=S p\left\{g_{1}, \cdots, g_{m}\right\} . \Delta$ is said to be strongly $(f, g)$-invariant at $p$ $\in M$ if there exist a neighborhood $U$ of $p, \alpha \in C_{m}^{\infty}(U)$ and $\beta \in G 1(m$, $C^{\infty}(U)$ ), such that on $U$

$$
\begin{gathered}
{[f+g \alpha, \Delta] \subset \Delta,} \\
{\left[(g \beta)_{i}, \Delta\right] \subset \Delta, \quad i=1, \cdots, m .}
\end{gathered}
$$

The local equivalence of these two kinds of $(f, g)$-invariances is proved in [6] and [12] independently.

\section{COMPATIBLE $(f, g)$-INVARIANCE}

To study decoupling problems of nonlinear systems, we have to consider several $(f, g)$-invariant distributions simultaneously. Thus, we introduce the concept of compatible $(f, g)$-invariance.

Definition 2.l: Let $\Delta_{1}, \cdots, \Delta_{k}$ be $k$ weakly $(f, g)$-invariant distributions at $p . \Delta_{1}, \cdots, \Delta_{k}$ are said to be compatible $(f, g)$-invariant at $p$, if there exist a neighborhood $U$ of $p, \alpha \in C_{m}^{\infty}(U)$ and $\beta \in G 1(m$, $\left.C^{\infty}(U)\right)$, such that on $U$

$$
\begin{gathered}
{\left[f+g \alpha, \Delta_{i}\right] \subset \Delta_{i}} \\
{\left[(g \beta)_{j}, \Delta_{i}\right] \subset \Delta_{i}, \quad j=1, \cdots, m ; i=1, \cdots, k .}
\end{gathered}
$$

Let $\Delta$ be an involutive distribution with constant dimension. According to Frobenius' theorem, there exists a local coordinate chart $(U,(x, y)), x$ $=\left(x_{1}, \cdots, x_{p}\right)$, and $y=\left(y_{1}, \cdots, y_{n-p}\right)$, such that

$$
\Delta=S p\left\{\frac{\partial}{\partial x_{i}}: \quad i=1, \cdots, p\right\}
$$

This coordinate chart is called a flat chart [10]. Let $\left(W,\left(x^{\prime}, y^{\prime}\right)\right)$ be another flat coordinate chart and $W \cap U \neq \phi$. Then on $W \cap U$

$$
y^{\prime}=y^{\prime}(y) \text {. }
$$

Assume a vector field $X$ is expressed in a flat chart $(x, y)$ as

$$
X=\left(a_{1}, \cdots, a_{n}\right)^{T} \in T(U) .
$$

Then the canonical projection $\pi(X)$ of $X$ on $T M / \Delta$ is defined as

$$
\pi(X)=\left(a_{p+1}, \cdots, a_{n}\right)^{T}
$$

and denoted as $X / \Delta$. Using (2.2), it is easy to prove that $X / \Delta$ is independent of the choice of the flat frame.

Likewise, for a distribution $G$ we may define the canonical projection 
$\pi(G)$ as

$$
G / \Delta=\pi(G)=\operatorname{Sp}\{\pi(X) \mid X \in G\}
$$

Now if $(x, y)$ is a flat chart, then $\left(\pi\left(\partial / \partial y_{1}\right), \cdots, \pi\left(\partial / \partial y_{n-p}\right)\right)$ is a basis of $T M / \Delta$. We say $G / \Delta$ has constant rank if $\pi$ restricted to $G$ has constant rank.

Now let $D_{0}, D_{1}, \cdots, D_{k}$ be $k+1$ simultaneously integrable distributions [11] and locally around $p \in U, \Sigma_{i=0}^{k} D_{i}=T(U)$. We construct $k$ distributions $\Delta_{1}, \cdots, \Delta_{k}$ as follows:

$$
\Delta_{i}=D_{0}+D_{i}, \quad i=1, \cdots, k .
$$

Consider the compatible $(f, g)$-invariance of $\Delta_{1}, \cdots, \Delta_{k}$ around the point $p$; we have the following theorem.

Theorem 2.2: Let $\Delta_{1}, \cdots, \Delta_{k}$ be $k$ distributions constructed as above. Assume $G / D_{0}$ is nonsingular at $p$ and

$$
G=G \cap \Delta_{1}+\cdots+G \cap \Delta_{k} .
$$

Then $\Delta_{1}, \cdots, \Delta_{k}$ are compatible $(f, g)$-invariant if and only if, each $\Delta_{i}$ is weakly $(f, g)$-invariant at $p$.

Proof: The necessity is trivial. We prove the sufficiency only. Choose local coordinates $x$, such that [11]

$$
D_{i}=S p\left\{\frac{\partial}{\partial x_{j}^{i}}: \quad j=1, \cdots, n_{i}\right\}, i=0,1, \cdots, k .
$$

Under this fixed coordinate frame we decompose $f$ and $g$ with respect to $D_{i}$ as

$$
\begin{gathered}
f=\left(\left(f^{0}\right)^{T},\left(f^{\mathrm{l}}\right)^{T}, \cdots,\left(f^{k}\right)^{T}\right)^{T}, \\
g_{j}=\left(\left(g_{j}^{0}\right)^{T},\left(g_{j}^{1}\right)^{T}, \cdots,\left(g_{j}^{k}\right)^{T}\right)^{T}, \quad j=1, \cdots, m .
\end{gathered}
$$

Set

$$
D^{i}=\sum_{j \neq i} D_{j}, \quad i=1, \cdots, k
$$

From (2.6), (2.7), and the fact that $G / D_{0}$ is nonsingular, one may see that $G / D^{i}$ is also nonsingular, $i=1, \cdots, k$. Thus,

$$
\operatorname{dim}\left(G / D^{i}\right):=t_{i} \leq \operatorname{dim}\left(G \cap \Delta_{i}\right) .
$$

Let $\widehat{B}_{i}$ be any basis of $G / D_{i}$. Fix flat coordinate frame $x ; B_{i}$ may be expressed as a matrix $B_{i}$. Then there exists (pointwise) a $t_{i} \times m$ matrix $E_{i}$ with rank $\left(E_{i}\right)=t_{i}$, such that

$$
\left(g_{1}^{i}, \cdots, g_{m}^{i}\right)=B_{i} E_{i}, \quad i=1, \cdots, k .
$$

It follows that

$$
G_{2}:=\left[\begin{array}{ccc}
g_{1}^{1} & & g_{m}^{l} \\
\vdots & \cdots & \vdots \\
g_{1}^{k} & & g_{m}^{k}
\end{array}\right]=\left[\begin{array}{ccc}
B_{1} & & \\
& \ddots & \\
& & B_{k}
\end{array}\right]\left[\begin{array}{c}
E_{1} \\
\vdots \\
E_{k}
\end{array}\right]:=B E .
$$

$E$ may be solved from (2.8) as

$$
E=\left(B^{T} B\right)^{-1} B^{T} G_{2}
$$

Thus, it is clear that $E$ is a $t \times m$ smooth matrix. Similarly, we may also express $B$ by $G_{2}$ as

$$
G_{2} E^{T}\left(E E^{T}\right)^{-1}=B
$$

Note that

$$
\operatorname{rank}(E)=\operatorname{rank}(B)=\sum_{i=1}^{k} t_{i}:=t \leq m
$$

Using this fact, one may find an $m \times(m-t)$ smooth matrix $Q$ such that

$$
\operatorname{rank}\left(Q, E^{T}\left(E E^{T}\right)^{-1}\right)=m \text {. }
$$

Set

$$
\begin{gathered}
W=Q-E^{T}\left(E E^{T}\right)^{-1} E Q, \\
L=E^{T}\left(E E^{T}\right)^{-1}, \\
\beta_{0}=(W, L) .
\end{gathered}
$$

It follows from (2.8) and (2.10) that

$$
g \beta_{0}=\left(\begin{array}{c|c}
X & X \\
0 & B_{1}+B_{2}+\cdots+B_{k}
\end{array}\right)
$$

where $X$ stands for some uncertain elements, + means direct sum of matrices. Next we denote

$$
B_{i}\left(x^{i}\right):=B_{i}\left(x_{p}^{0}, \cdots, x_{p}^{i-1}, x^{i}, x_{p}^{i+1}, \cdots, x_{p}^{k}\right),
$$

i.e., set all block coordinates $x^{j}$, except $x^{i}$, to be $x_{p}^{j}$, which is the $j$ th block coordinates of the point $p$. Then $B_{i}^{T}\left(x^{i}\right) B_{i}(x)$ is locally nonsingular because it is nonsingular at $p$. Setting

$$
\beta_{i}=\left(B_{i}^{T}\left(x_{i}\right) B_{i}(x)\right)^{-1}, \quad i=1, \cdots, k
$$

we construct $\alpha$ and $\beta$ as follows:

$$
\alpha=-L\left[\begin{array}{ccc}
\beta_{1} & & \\
& \ddots & \\
& & \beta_{k}
\end{array}\right] \underset{\left(D^{T} D\right)^{-1} D^{T}}{\beta=\beta_{0}\left(I_{m-1}+\beta_{1}+\cdots+\beta_{k}\right)}\left[\begin{array}{c}
f^{1} \\
\vdots \\
f^{k}
\end{array}\right]
$$

where $L$ and $\beta_{0}$ are constructed by (2.10) and

$$
D=\left(B_{1} \beta_{1}+\cdots+B_{k} \beta_{k}\right) \text {. }
$$

Using the canonical form (2.7) of $D_{i}$ and the fact that $\Delta_{i}$ 's are weakly ( $f$, $g$ )-invariant, one may verify that the above $\alpha$ and $\beta$ satisfy (2.1).

In the above proof, the feedback law $(\alpha, \beta)$ and a decoupling form have been constructed carefully. It will be used later on.

\section{NONINTERACTING DECOMPOSITION THEOREM}

Consider the nonlinear system (1.1) again. The noninteracting decomposition problem is described precisely as follows.

Definition 3.1: Given a partition of the output (1.1.b) as

$$
y=\left\{y^{1}, \cdots, y^{k}\right\}:=\left\{h^{1}, \cdots, h^{k}\right\}
$$

the noninteracting decomposition problem (NDP) is solvable around $p \in$ $M$, if there exists a neighborhood $U$ of $p, \alpha \in C_{m}^{\infty}(U)$ and $\beta \in G \mathrm{I}(\mathrm{m}$, $\left.C^{\infty}(U)\right)$, such that by using the feedback control $u=\alpha(x)+\beta(x) v$, there exists a partition of the input $v$, say $v^{0}, v^{1}, \cdots, v^{k}$, such that i) $v^{i}$ does not affect $y^{j}(j \neq i)$, and ii) $v^{i}$ completely controls $y^{i}, i=1,2, \cdots$, $k$.

Next we give some notations and conventions. Denote

$$
K_{i}:=\bigcap_{j \neq i} \operatorname{ker}\left(h_{*}^{j}\right), \quad i=1, \cdots, k
$$

Let $W_{i}$ be the largest weakly $(f, g)$-invariant distribution contained in $K_{i}$, and $R_{i}$ the largest controllability distribution in $K_{i}$. Set $R=\sum_{i=1}^{k} R_{i} ; W$ $=\Sigma_{i=1}^{k} W_{i} ; H_{i}=\Sigma_{j \neq i} W_{j}$; and $\bar{H}_{i}$ : the involutive closure of $H_{i}$, i.e., the smallest involutive distribution containing $H_{i}$.

In order to state and prove the theorem of NDP, a few preliminary results are necessary.

Lemma 3.2 [3]: Assume the NDP is solvable, then

$$
G=G \cap R_{1}+\cdots+G \cap R_{k}
$$

Lemma 3.3 [3]: Assume the system (1.1) is strongly accessible [14], and $G=G \cap R_{1}+\cdots+G \cap R_{k}$. Then $R=T(U)$, i.e., locally dim $(R)=n$.

Lemma 3.4: Let $\Delta_{1}$ and $\Delta_{2}$ be two weakly $(f, g)$-invariant distribu- 
tions, $G=G \cap \Delta_{1}+G \cap \Delta_{2}$. Then their intersection $\Delta_{1} \cap \Delta_{2}$ is another weakly $(f, g)$-invariant distribution.

Proof: Let $X \in \Delta_{1} \cap \Delta_{2}$, then there exist $Y_{1} \in \Delta_{1}, Y_{2} \in \Delta_{2}$, and $g_{1}, g_{2} \in G$, such that

$$
[f, X]=Y_{1}+g_{1}=Y_{2}+g_{2} .
$$

Thus,

$$
Y_{1}-Y_{2}=g_{2}-g_{1} \in G
$$

Since $G=G \cap \Delta_{1}+G \cap \Delta_{2}$, there exist $g_{1}^{\prime}$ and $g_{2}^{\prime}$, such that $g_{1}^{\prime} \in$ $G \cap \Delta_{1}, g_{2}^{\prime} \in G \cap \Delta_{2}$, and $Y_{1}-Y_{2}=g_{1}^{\prime}+g_{2}^{\prime}$. Set

$$
Y_{3}:=Y_{1}-g_{1}^{\prime}=Y_{2}+g_{2}^{\prime} \in \Delta_{1} \cap \Delta_{2} \text {. }
$$

Then

$$
[f, X]=Y_{3}+g_{1}^{\prime}+g_{1} \in \Delta_{1} \cap \Delta_{2}+G,
$$

i.e.,

$$
\left[f, \Delta_{1} \cap \Delta_{2}\right] \subset \Delta_{1} \cap \Delta_{2}+G
$$

Similarly

$$
\left[g_{i}, \Delta_{1} \cap \Delta_{2}\right] \subset \Delta_{1} \cap \Delta_{2}+G .
$$

Remark 3.5: Since $R_{i} \subset W_{i}$, it follows that

$$
\begin{gathered}
W=\sum_{i=1}^{k} W_{i}=T(U), \\
G=G \cap W_{1}+\cdots+G \cap W_{k} .
\end{gathered}
$$

Now we are ready to present the following theorem which solves NDP. Theorem 3.6: Assume that the affine nonlinear system (1.1) has strong accessibility property and all $\vec{H}_{i}, i=1, \cdots, k$ as well as $G /\left(\Sigma_{i=1}^{k} W_{i} \cap\right.$ $\bar{H}_{i}$ ) are nonsingular. Then the NDP is solvable if and only if

$$
G=G \cap W_{1}+\cdots+G \cap W_{k} .
$$

Note that there is an "illegal" use of the notation $G /\left(\sum_{i=1}^{k} W_{i} \cap \bar{H}_{i}\right)$ in the Theorem's statement because we do not know whether $\Sigma_{i=1}^{k} W_{i} \cap \bar{H}_{i}$ is nonsingular and involutive or not. But in the following proof, Lemma 3.7 will show its legality.

Proof: The necessity follows from Lemma 3.2 and Remark 3.5. Thus, we have only to prove the sufficiency.

According to Frobenius' theorem, we can find a set of $C^{\infty}$ functions $z^{i}$ $=\left(z_{1}^{i}, \cdots, z_{n_{i}}^{i}\right)$, where $n_{i}=n-\operatorname{dim}\left(\bar{H}_{i}\right)$, such that

$$
\bar{H}_{i}^{1}=S p\left\{d z_{j}^{i}: \quad j=1, \cdots, n_{i}\right\}, i=1, \cdots, k .
$$

We claim that the codistributions $\Omega_{i}:=\bar{H}_{i}^{\perp}$ are linearly independent. Assume there exists a $\omega_{i} \in \Omega_{i}$ such that $\omega_{i}=\Sigma_{j \neq i} a_{j} \omega_{j}$, where $\omega_{j} \in \Omega_{j}$. Then

$$
\omega_{i} \in \bar{\sum}_{j \neq i} W_{j}^{\perp} \subset\left(\sum_{j \neq i} W_{j}\right)^{\perp}
$$

and

$$
\omega_{i} \in W_{i}^{\perp},
$$

i.e.,

$$
\omega_{i} \in W^{\perp}=T(U)^{\perp}=0 .
$$

Thus, we can choose $z^{0}=\left(z_{1}^{0}, \cdots, z_{n_{0}}^{0}\right)$, such that $\Sigma_{i=0}^{k} n_{i}=n$ and $\left(z^{0}, z^{1}, \cdots, z^{k}\right)$ forms a local coordinate frame. Now the original system may be expressed in the $z$ coordinates as

$$
\dot{z}=f(z)+g(z) u .
$$

We will decompose it with respect to coordinates $z$. Thus, we define a set of distributions by $z$ coordinates as

$$
D_{i}=S p\left\{\frac{\partial}{\partial z_{j}^{i}}: \quad j=1, \cdots, n_{i}\right\}, \quad i=0,1, \cdots, k .
$$

Under $z$ coordinates, it is clear that

$$
d z_{s}^{j} \in \bar{H}_{j}^{\perp} \subset W_{i}^{\perp}, \quad j=1, \cdots, k ; s=1, \cdots, n_{j} ; j \neq i .
$$

Thus, we have

$$
\left\langle d z_{s}^{j}, W_{i}\right\rangle=0, \quad j \neq i
$$

It follows that

$$
W_{i} \subset D_{0}+D_{i}, \quad i=1, \cdots, k
$$

Moreover,

$$
H_{i}=\sum_{j \neq i} W_{j} \subset \sum_{j \neq i} D_{j}, \quad i=1, \cdots, k
$$

Since the right-hand side of (3.6) is involutive, it follows that

$$
\bar{H}_{i} \subset \sum_{j \neq i} D_{j}, \quad i=1, \cdots, k
$$

To reveal the relations between $W_{i}, \bar{H}_{i}, i=1, \cdots, k$ and the canonical distributions $D_{i}, i=0, \cdots, k$, we prove the following lemmas.

Lemma 3.7:

$$
D_{0}=\sum_{i=1}^{k} W_{i} \cap \bar{H}_{i}
$$

Proof: Let $X \in D_{0}$, since $W_{1}+\cdots+W_{k}=T(U)$, there exist $X_{i}$ $\in W_{i}, i=1, \cdots, k$, such that $X=X_{1}+\cdots+X_{k}$. Using (3.16), we have

$$
\left\langle d z_{s}^{j}, X\right\rangle=\left\langle d z_{s}^{j}, X_{j}\right\rangle, \quad j=1, \cdots, k .
$$

But $X \in D_{0}$, it follows that $\left\langle d z_{s}^{j}, X_{j}\right\rangle=0$. Thus,

$$
X_{j} \in\left(\Omega_{j}\right)^{\perp}=\bar{H}_{j} .
$$

Conversely, if $X \in \Sigma_{i=1}^{k} W_{i} \cap \bar{H}_{i}$, then there exist $X_{i} \in W_{i} \cap \bar{H}_{i}$, $i=1, \cdots, k$, such that $X=X_{1}+\cdots+X_{k}$. Since $X_{i} \in \bar{H}_{i}=$ $\Omega_{i}^{\perp},\left\langle d z_{s}^{i}, X_{i}\right\rangle=0$. Since $X_{i} \in W_{i}$ too, $\left\langle d z_{s}^{j}, X_{i}\right\rangle=0, j \neq i$. Then it is clear that $X_{i} \in D_{0}, i=1, \cdots, k$, and so is $X$.

Lemma 3.8:

$$
D_{0}+D_{i}=W_{i}+\sum_{j=1}^{k}\left(W_{j} \cap \bar{H}_{j}\right), \quad i=1, \cdots, k
$$

Proof: Equations (3.5) and (3.8) imply that the right-hand side of (3.9) is contained on the left-hand side.

Conversely, if $X \in D_{i}$, as in the proof of Lemma 3.7, there exist $X_{i} \in$

$W_{i}, i=1, \cdots, k$, such that $X=X_{1}+\cdots+X_{k}$. Then

$$
\left\langle d z_{s}^{j}, X\right\rangle=\left\langle d z_{s}^{j}, X_{j}\right\rangle \text {. }
$$

But since $X \in D_{i}$, it is clear that

$$
\left\langle d_{s}^{j}, X_{j}\right\rangle=0, \quad j \neq i .
$$

Thus, $X_{j} \in D_{0}, j \neq i$, and hence $X \in D_{0}+W_{i}$, i.e., $D_{i} \subset D_{0}+W_{i}$. The conclusion follows from Lemma 3.7 immediately.

Now we continue the proof of Theorem 3.6.

First, we claim that $D_{0}+D_{i}$ is weakly $(f, g)$-invariant. It is easy to see that the summation of several weakly $(f, g)$-invariant distributions is another weakly $(f, g)$-invariant distribution. Thus, $H_{j}$ is weakly $(f, g)$ invariant, and so is $\bar{H}_{j}$, the involutive closure of $H_{j}$ [9]. According to Lemma 3.8, to prove that $D_{0}+D_{i}$ is weakly $(f, g)$-invariant it is enough 
to show that $W_{j} \cap \bar{H}_{j}$ is weakly $(f, g)$-invariant. Since $G=G \cap W_{1}+$ $\cdots+G \cap W_{k} \subset G \cap W_{j}+G \cap \bar{H}_{j}$, it follows that $G=G \cap W_{j}+$ $G \cap \bar{H}_{j}$. Using Lemma 3.4 , the claim is obviously true.

As in the above, one may also see that

$$
G=G \cap W_{1}+\cdots+G \cap W_{k}=G \cap\left(D_{0}+D_{1}\right)+\cdots+G \cap\left(D_{0}+D_{k}\right) .
$$

Now it can be readily verified that for $D_{0}+D_{i}, i=1, \cdots, k$, all the requirements of Theorem 2.2 are satisfied. Thus, we can construct $\alpha$ and $\beta$, as in the proof of Theorem 2.2, such that

$$
\left[f+g \alpha, D_{0}+D_{i}\right] \subset D_{0}+D_{i}
$$

$\left[(g \beta)_{j}, D_{0}+D_{i}\right] \subset D_{0}+D_{i}, \quad j=1, \cdots, m ; i=1, \cdots, k$.

According to (3.10) and the canonical form (3.4) of $D_{i}$, a straightforward computation shows that the feedback system $\dot{z}=f+g \alpha+g \beta v$ has the following decomposed form:

$$
\begin{aligned}
& \dot{z}^{0}=f^{0}(z)+g^{0}(z) v \\
& \dot{z}^{1}=f^{1}\left(z^{1}\right)+g^{1}\left(z^{1}\right) v^{1} \\
& \cdots \\
& z^{k}=f^{k}\left(z^{k}\right)+g^{k}\left(z^{k}\right) v^{k} .
\end{aligned}
$$

Now it is obvious that $v^{i}$ does not affect $z^{j}, j=1, \cdots, k, j \neq i$. To show that $v^{i}$ does not affect $h^{j}$, it is enough to show that $h^{j}=h^{j}\left(z^{j}\right)$. Since $W_{j} \subset \operatorname{ker}\left(h_{*}^{i}\right)=\left(S p\left\{d h^{i}\right\}\right)^{\perp}, j \neq i$, it follows that

$$
\left(S p\left\{d h^{i}\right\}\right)^{\perp} \supset H_{i} .
$$

Note that the left-hand side of the above equation is involutive, thus

$$
\text { . }\left(S p\left\{d h^{i}\right\}\right)^{\perp} \supset \bar{H}_{i} \text {. }
$$

It follows that

$$
d h^{i} \in \bar{H}_{i}^{\perp}=\Omega_{i}=S p\left\{d z_{j}^{i}: \quad j=1, \cdots, n_{i}\right\}
$$

which means that

$$
h^{j}=h^{j}\left(z^{j}\right), \quad j=1, \cdots, k .
$$

To see that $v^{i}$ completely controls $h^{i}$, a simple computation shows that

$$
\left.\left.R_{i}\right|_{z} \subset D_{i}\right|_{z}, \quad z \in U, i=1, \cdots, k .
$$

Using this fact and the equation (3.13), one may easily see that

$$
\operatorname{rank}\left(J_{h^{i}}\right)=\operatorname{dim}\left(h_{*}^{i}\left(R_{i}\right)\right)
$$

where $J_{h^{i}}$ is the Jacobian matrix of $h^{i}$. Thus, $v^{i}$ controls $h^{i}$ completely. $\square$

\section{NONINTERACTING FORM}

Summarizing the previous constructive proofs, we obtained the following algorithm.

Algorithm 4.1

Step I: Verify that the system is strong accessible.

Step 2: Compute $K_{i}:=\bigcap_{j \neq i} \operatorname{ker}\left(h_{*}^{j}\right)$ and $\dot{W}_{i}$, the largest weakly $(f$, $g$ )-invariant distribution contained in $K_{i}$.

Step 3: Set

$$
H_{i}=\sum_{j \neq i} W_{j}, \quad i=1, \cdots, k .
$$

Compute $\breve{H}_{i}$ and verify that $\tilde{H}_{i}$ 's are nonsingular.

Step 4: Check that

$$
G=G \cap W_{1}+\cdots+G \cap W_{k} .
$$

Step 5: Choose coordinates $z=\left(z_{1}^{0}, \cdots, z_{n_{0}}^{0} ; z_{1}^{1}, \cdots, z_{n_{1}}^{1} ; \cdots ; z_{1}^{k}\right.$, $\cdots, z_{n_{k}}^{k}$ ) such that

$$
\bar{H}_{i}^{\perp}=S p\left\{d z_{j}^{i}: \quad j=1, \cdots, n_{i}\right\}, \quad i=1, \cdots, k .
$$

Step 6: Choose any basis $B_{i}$ of $G / D^{i}$ where

$$
\begin{gathered}
D_{i}=S p\left\{\frac{\partial}{\partial z_{j}^{i}}: \quad j=1, \cdots, n_{i}\right\}, \quad i=0,1, \cdots, k . \\
D^{i}=\sum_{j \neq i} D_{j} .
\end{gathered}
$$

Step 7: Follow the procedure of (2.8)-(2.13) to construct the feedback law $\alpha, \beta$, and the feedback control system

$$
\begin{gathered}
\dot{z}=f(z)+g(z) \alpha+g(z) \beta v, \\
y=h(z) .
\end{gathered}
$$

The following theorem shows that the above Algorithm is workable.

Theorem 4.2: Under the assumptions of Theorem 3.6, the NDP is solvable if and only if, Algorithm 4.1 is executible and under the coordinates $z$ the feedback control system (4.1) has the following noninteracting form:

$$
\begin{aligned}
& \dot{z}^{0}=f^{0}(z)+g^{0}(z) v \\
& \dot{z}^{1}=f^{1}\left(z^{1}\right)+g^{1}\left(z^{1}\right) v^{1} \\
& \quad \cdots \\
& \dot{z}^{k}=f^{k}\left(z^{k}\right)+g^{k}\left(z^{k}\right) v^{k}, \\
& y_{i}=h^{i}\left(z^{i}\right), \quad i=1, \cdots, k .
\end{aligned}
$$

\section{6 .}

Proof: It follows from the constructive proofs of Theorems 2.2 and

To reveal the physical meaning of the decomposed substates of (4.2), let us turn back to the proof of Theorem 3.6. By (3.11) we have $W_{i} \subset D_{0}$ $+D_{i}$. Clearly, $D_{0}+D_{i} \subset \cap_{j \neq i} \operatorname{ker} h_{*}^{j}$ and $D_{0}+D_{i}$ is $(f, g)$-invariant. Hence, $D_{0}+D_{i}=W_{i}$ is the largest weakly $(f, g)$-invariant distribution contained in $\bigcap_{j \neq i}$ ker $h_{*}^{j}$. Moreover, from (4.2) it is also clear that $D_{0}$ is the largest weakly $(f, g)$-invariant distribution contained in $\cap_{j=1}^{k} \operatorname{ker} h_{*}^{j}$. The decomposed form (4.2), therefore, has significant physical meaning.

Using (3.9), (4.2) shows that $D_{0}+D_{i}, i=1, \cdots, k$ are compatible ( $f, g$ )-invariant. In [3] the controllability distributions $R_{i}$ are shown to be compatible $(f, g)$-invariant. Now we prove the following.

Proposition 4.3: Under the assumptions of Theorem 3.6 the largest weakly $(f, g)$-invariant distributions $W_{i} \subset \cap_{j \neq i}$ ker $h_{*}^{j}$ are compatible $(f, g)$-invariant.

The rest of this section will be devoted to showing that the noninteracting decomposed form (4.1) is a canonical form.

A coordinate frame $z=\left(z_{1}^{0}, \cdots, z_{n_{0}}^{0} ; z_{1}^{1}, \cdots, z_{n_{1}}^{1} ; \cdots ; z_{1}^{k}, \cdots, z_{n_{k}}^{k}\right)$ is called a flat coordinate frame with respect to $\bar{H}_{1}, \cdots, \bar{H}_{k}$, if

$$
\bar{H}_{i}{ }^{+}=s p\left\{d z_{j}^{i}: \quad j=1, \cdots, n_{i}\right\}, \quad i=1, \cdots, k .
$$

In the noninteracting decomposed system (4.1), we call each subsystem

$$
\begin{gathered}
z^{i}=f^{i}\left(z^{i}\right)+g^{i}\left(z^{i}\right) v^{i} \\
y^{i}=h^{i}\left(z^{i}\right), \quad i=1, \cdots, k
\end{gathered}
$$

the input-output noninteracting subsystem.

The noninteracting form (4.2) is a canonical form in the following sense.

Theorem 4.3: Let $x$ be another flat coordinate frame with respect to $\bar{H}_{1}, \cdots, \bar{H}_{k}$. If under the coordinates $x$, one obtained another noninteracting decomposed form

$$
\begin{aligned}
& \dot{x}^{0}=\bar{f}^{0}(x)+\bar{g}^{0}(x) w \\
& \dot{x}^{1}=\bar{f}^{1}\left(x^{1}\right)+\bar{g}^{1}\left(x^{1}\right) w^{1} \\
& \cdots \\
& \dot{x}^{k}=\bar{f}^{k}\left(x^{k}\right)+\bar{g}^{k}\left(x^{k}\right) w^{k} \\
& y_{i}=\bar{h}^{i}\left(x^{i}\right), \quad i=1, \cdots, k
\end{aligned}
$$

where $\tilde{g}^{i}$ has full rank, $i=1, \cdots, k$. Comparing it to (4.2), there exist 
substate diffeomorphisms $F_{i}: z^{i} \rightarrow x^{i}, i=1, \cdots, k$; and substate feedback laws $\alpha_{i}\left(x^{i}\right), \beta_{i}\left(x^{i}\right), i=1, \cdots, k$, such that

$$
\begin{gathered}
\bar{f}^{i}=\left(F_{i}\right)_{*} f^{i}+\left(F_{i}\right)_{*} g^{i} \alpha_{i} \\
\bar{g}^{i}=\left(F_{i}\right)_{*} g^{i} \beta_{i}, \\
h^{i}=\left(F_{i}^{-1}\right)^{*} h .
\end{gathered}
$$

That is, each input-output noninteracting subsystem is unique, modulo a subsystem substate diffeomorphism, and a subsystem substate feedback control.

\section{ACKNOWLEDGMENT}

The author is indebted to Prof. T. J. Tarn and Prof. A. Isidori for useful conversations and much help.

\section{REFERENCES}

[1] W. M. Wonham, Linear Multivariable Control: A Geometric Approach. New York: Springer-Verlag, 1979.

[2] A. S. Morse and W. M. Wonham, "Status of noninteracting control," IEEE Trans. Automat. Contr., vol. AC-16, pp. 568-581, 1979.

[3] H. Nijmeijer and J. M. Schumacher, "The regular local noninteracting control problem for nonlinear control systems," SIAM J. Contr. Optimiz., vol. 24, no. problem for nonlinear cont
6 , pp. $1232-1245,1986$.

[4] - , "Zeros at infinity for affine nonlinear control systems," IEEE Trans. Automat. Contr., vol. AC-30, no. 6, pp. 566-573, 1985.

[5] R. Hirschorn, "(A, B)-invariant distributions and disturbance decoupling of nonlinear systems," SIAM J. Contr. Optimiz., vol. 19, pp. 1-19, 1981.

[6] A. Isidori, A. J. Krener, C. Gori-Fiorgi, and S. Monaco, "Local $(f, g)$-invariant distributions," Syst. Contr. Lett., vol. 1, pp. 12-15, 1981.

[7] A. J. Krener and A. Isidori, (adf, G)-Invariant and Controllability Distributions (Lecture Notes in Control and Information Sciences, Vol. 39.) 1982, pp. 157-164.

[8] H. Nijmeijer, "Controllability distributions for nonlinear systems," Syst. Contr. Lett., vol. 2, pp. 122-129, 1982

[9] A. Isidori, Nonlinear Control Systems: An Introduction (Lecture Notes in Control and Information Sciences, Vol. 71). New York: Springer-Verlag, 1985.

[10] B. L. Reinhart, "Foliated manifold with bundle-like metrics," Annals Math., vol. 69 , no. $1,1959$.

[11] W. Respondek, "On decomposition of nonlinear control systems," Syst. Contr. Lett., vol. 1, pp. 301-308, 1982.

[12] H. Nijmeijer, "Controlled invariance for affine control systems," Int. J. Contr., vol. 34, pp. 824-833, 1981.

[13] W. M. Boothby, An Introduction to Differentiable Manifolds and Riemannian Geometry. New York: Academic, 1979.

[14] R. Hermann and A. J. Krener, "Nonlinear controllability and observability," IEEE Trans. Automat. Contr., vol. AC-22, no. 5, pp. 728-740, 1977.

\section{Low-Resolution Implementation of High-Resolution Position Control}

\section{HERBERT HANSELMANN}

\begin{abstract}
Digital implementation of position or path control using high-resolution encoders may require high resolution for control algorithm computations too if the controller state variables carry highprecision absolute position information. If the control algorithm is reformulated in a form in which the controller state is continuously offset according to the possibly nonconstant setpoint, then much less arithmetic wordlength may be sufficient.
\end{abstract}

\section{INTRODUCTION}

State-of-the-art high-precision position control systems may easily be equipped with digital position encoders yielding 18 or more bits. A

Manuscript received March 17, 1987; revised July 7, 1987.

The author was with the Department of Automatic Control in Mechanical Engineering, University of Paderborn, Paderborn, West Germany. He is now the dSPACE Digital Signal Processing and Control Engineering GmbH, Paderborn, West Germany. IEEE Log Number 8822746. position signal spanning a range of $2 \mathrm{~m}$, for instance, is then quantized with about $8 \mu \mathrm{m}$ resolution, and this resolution may indeed be necessary to achieve the design goals, as in the example given in Section IV.

Usually, implementation of position control based on such position sensor signals directly requires arithmetic resolution even higher than the sensor resolution. This may be in conflict with the capabilities of some target processors (such as digital signal processors [1], [2]), but is undesirable at least whenever the standard wordlength supported is exceeded.

Fig. 1 shows a typical control system. It performs path control, i.e., the position reference signal is usually not constant. In robotics it is common to feedforward velocity and acceleration reference signals in addition to the position reference in order to minimize path-following errors. The system in the dash-lined box is not part of the final control system, but models the feedforward signals during design. Besides the position there may be several auxiliary measurement signals, some of which may also be high resolution position signals. All signals are collected in the measurement vector $y_{m}$.

The design of the controller may be performed according to the structure from Fig. 1. The problem, however, is that in this case the absolute position will be represented in the state variables of the controller, and, typical of most practical position control applications, full control action will be produced for position errors many orders of magnitude smaller than the absolute position range. Since the full absolute position which may be accurate to, say, 18 bits must be represented within the limited wordlength of the target processor arithmetic, but only a few least significant bits are responsible for producing the control signal, there may be severe quantization effects or else the wordlength demand becomes excessive.

This situation can be alleviated by implementing the control in an error form as sketched in Fig. 2 (the error signals are $e_{r}$ and $e_{a}$ ), along with a nonconstant controller state variable offset leading to a reformulated controller. It is the purpose of this note to present a systematic technique to eliminate the absolute position information from the state variables of the controller. The controller state variables will then be zero in any stationary position, and the arithmetic precision available can be fully used for the transient deflections which produce the control action. Eliminating absolute position information is particularly important when short wordlength fixed point arithmetic (say 16 bits) has to be used. But note that even a standard 32 bit floating point format accommodates only 24 bits in the mantissa, so the problem at hand may also be of importance in this case.

Using position error signals in the control algorithm has always been the standard technique with conventional cascade type position control systems. For other types of compensators such as those derived via optimal control theory, an appropriate technique does not seem to be available. The problem discussed has some connection with the constant nonzero setpoint issue as discussed, for instance, in [3], but note that we do not stick to a particular controller configuration such as state-feedback, nor do we restrict the reference signal to being constant.

The proposed reformulation technique can be applied both to a continuous controller which later on might be discretized, or to an already discrete controller, be it designed in the discrete domain or be it a discretized version of a continuous controller. The continuous case, which already shows the main ideas, is considered first in Section II. The discrete case is a little bit more involved and requires separate discussion which is given in Section III. For clarity, all derivations are made for the situation shown in Figs. 1 and 2, but can easily be adapted to different sensor signal situations. The technique is illustrated by an industrial application example in Section IV.

\section{REFORMULATION OF A CONTINUOUS CONTROLlER}

Let the original controller be given in state-space form as

$$
\dot{x}=A x+b_{y} y+b_{r} r+b_{a} y_{a}+\sum_{\nu \in S_{o}} b_{\nu} y_{m, \nu}+b_{r} \dot{r}+b_{\dot{r}} \dot{r}
$$

